\title{
mTOR Hyperactivity Levels Influence the Severity of Epilepsy and Associated Neuropathology in an Experimental Model of Tuberous Sclerosis Complex and Focal Cortical Dysplasia
}

\author{
늘 H. Nguyen, ${ }^{1}$ Travorn Mahadeo, ${ }^{1}$ and $\complement^{-A n g e ́ l i q u e ~ B o r d e y ~}{ }^{1,2}$ \\ ${ }^{1}$ Department of Neurosurgery, and ${ }^{2}$ Department of Cellular and Molecular Physiology, Yale University School of Medicine, New Haven, Connecticut 06520
}

Tuberous sclerosis complex (TSC) and focal cortical dysplasia (FCD) are focal malformations of cortical development (FMCDs) that are highly associated with intractable epilepsy. TSC and FCD are mTORopathies caused by a spectrum of pathogenic variants in the mechanistic target of rapamycin (mTOR) pathway genes leading to differential activation of mTOR signaling. However, whether the degree of mTOR hyperactivity influences disease severity remains unclear. Here, we examined the effects of differential mTOR hyperactivity levels on epilepsy and associated neuropathology in a mouse model of TSC and FCD. Constitutively active Rheb (Rheb ${ }^{\mathrm{CA}}$ ), the canonical activator of mTOR complex 1 (mTORC1), was expressed in mouse embryos of either sex via in utero electroporation at low, intermediate, and high concentrations to induce different mTORC1 activity levels in developing cortical neurons. We found that Rheb ${ }^{\mathrm{CA}}$ expression induced $\mathrm{mTORC1}$ hyperactivation and increased neuronal soma size and misplacement in a dose-dependent manner. No seizures were detected in the low Rheb ${ }^{\mathrm{CA}}$ mice, whereas the intermediate and high Rheb ${ }^{\mathrm{CA}}$ mice displayed spontaneous, recurrent seizures that significantly increased with higher Rheb ${ }^{\mathrm{CA}}$ concentrations. Seizures were associated with a global increase in microglial activation that was notably higher in the regions containing $\mathrm{Rheb}^{\mathrm{CA}}$-expressing neurons. These data demonstrate that neuronal mTOR hyperactivity levels influence the severity of epilepsy and associated neuropathology in experimental TSC and FCD. Overall, these findings highlight the importance of evaluating the outcome of individual variants on mTOR activity levels and support personalized medicine strategies based on patient variants and mTOR activity level for TSC, FCD, and potentially other mTORopathies.

Key words: epilepsy; focal cortical dysplasia; microglia; mTOR; personalized medicine; tuberous sclerosis complex

Significance Statement

Tuberous sclerosis complex (TSC) and focal cortical dysplasia (FCD) are epileptogenic cortical malformations caused by pathogenic variants in mechanistic target of rapamycin (mTOR) pathway genes leading to differential mTOR hyperactivation. Here, we present novel findings that neuronal mTOR hyperactivity levels correlate with the severity of epilepsy and associated neuropathology in a mouse model of TSC and FCD. Our findings suggest the need to evaluate the outcome of individual variants on mTOR activity levels in clinical assessments and support personalized medicine strategies based on patient variants and mTOR activity level. Additionally, we present useful modifications to a previously described mouse model of TSC and FCD that allows for titration of seizure frequency and generation of a mild to severe epilepsy phenotype as applicable for preclinical drug testing and mechanistic studies.

\section{Introduction}

Tuberous sclerosis complex (TSC) and focal cortical dysplasia (FCD) are neurodevelopmental disorders characterized by focal

Received Sept. 2, 2018; revised Jan. 14, 2019; accepted Jan. 15, 2019.

Author contributions: L.H.N. wrote the first draft of the paper; A.B. edited the paper; L.H.N. and A.B. designed research; L.H.N. performed research; L.H.N. and T.M. analyzed data; L.H.N. wrote the paper.

This work was supported by the National Institute of Neurological Disorders and Stroke-National Institutes of Health (Grant NS086329 to A.B.), the American Epilepsy Society Postdoctoral Fellowship (to L.H.N.), and the Brown Coxe Postdoctoral Fellowship (to L.H.N).

The authors declare no competing financial interests. malformations of cortical development (FMCDs) that are highly associated with epilepsy and cognitive impairments (Leventer et al., 2008). TSC- and FCD-related epilepsies are often intractable to medical therapy and account for the majority of childhood epilepsy cases requiring surgical treatment (Harvey et al., 2008; Blümcke et al., 2017). FMCDs in TSC, known as tubers, and in

Correspondence should be addressed to Angelique Bordey at angelique.bordey@yale.edu. https://doi.org/10.1523/JNEUROSCI.2260-18.2019

Copyright $\odot 2019$ the authors 
FCD, particularly type II (FCDII), share a number of neuropathological features, including cortical dyslamination due to neuronal misplacement; the presence of enlarged, dysmorphic neurons; glial activation; and enhanced mechanistic target of rapamycin (mTOR) signaling (Aronica et al., 2012; Vezzani et al., 2012; Crino, 2015). The mTOR pathway is a key regulator of cell growth and development and aberrant mTOR signaling is implicated in the cellular and molecular changes leading to cortical malformations and epilepsy in TSC and FCD (Lipton and Sahin, 2014).

TSC tubers and FCDII are caused by brain somatic (with or without germline) mutations in regulators of the mTOR pathway, leading to constitutive mTOR hyperactivation (Crino, 2015; Marsan and Baulac, 2018). TSC tubers result from mutations in TSC1 or TSC2 (Orlova and Crino, 2010), whereas FCDII has more recently been linked to mutations in MTOR, DEPDC5, NPRL2, NPRL3, PIK3CA, PTEN, TSC1, and TSC2 (Marsan and Baulac, 2018). More than 2500 pathogenic variants in TSC1 and TSC2 and numerous variants in other MTOR pathway genes have been identified to date (Northrup et al., 1999; Marsan and Baulac, 2018). Different variants can lead to different clinical phenotypes, with some patients presenting with few signs and symptoms and others exhibiting severe manifestations (Curatolo et al., 2015; Iffland and Crino, 2016; Rosset et al., 2017). The broad phenotypic spectrum in TSC and FCD can be attributed to differences in the developmental time points at which the mutations occur, the proportion of cells with the mutations, or the outcome of the gene variants on mTOR activity. Indeed, data from studies in patient tissue and cell lines suggest that distinct variants may confer different degrees of mTOR pathway activation (Nellist et al., 2005; Grabiner et al., 2014; Lim et al., 2015, 2017; Mirzaa et al., 2016). However, to our knowledge, no studies have examined the relationship between cellular mTOR activity levels and the severity of TSC and FCD phenotypes. Although mTOR hyperactivation in animal models consistently recapitulates human TSC and FCD features, including neuronal hypertrophy, cortical dyslamination, and epilepsy (Wong and Roper, 2016), it is unknown whether this is an all-or-nothing occurrence in which mTOR hyperactivation induces the phenotypes after reaching a certain threshold or a graded event in which increasing mTOR activity levels leads to progressively worsening phenotypes. Given that the mTOR pathway is an emerging therapeutic target for TSCand FCD-related epilepsy, understanding how cellular levels of mTOR hyperactivity influence disease outcome may be crucial for establishing optimal treatment strategies.

In this study, we investigated whether there is a correlation between mTOR hyperactivity levels and the severity of disease phenotypes using experimental FMCDs recapitulating TSC tubers and FCDII in mice. In this model, a constitutive active Rheb (Rheb ${ }^{\mathrm{CA}}$ ), the canonical activator of mTOR complex 1 (mTORC1), was expressed in developing cortical neurons via in utero electroporation to mimic persistent mTOR activation in disease states. By using different concentrations of Rheb ${ }^{\mathrm{CA}}$ plasmids, we were able to induce different levels of mTOR hyperactivation in a dose-dependent manner. We found that increasing mTOR hyperactivity levels caused a corresponding increase in neuronal soma size and misplacement. Additionally, increased mTOR hyperactivity levels were associated with higher seizure frequencies. These findings highlight the importance of evaluating individual gene variants in the context of mTOR activity levels and support personalized medicine strategies based on patient variants and mTOR activity level.

\section{Materials and Methods}

Animals. All animal procedures were performed in accordance with Yale University Institutional Animal Care and Use Committee's regulations. All experiments were performed on CD-1 mice of either sex (Charles River Laboratories).

In utero electroporation and DNA plasmids. Timed-pregnant embryonic day $15(\mathrm{E} 15)( \pm 0.5)$ mice were anesthetized with isoflurane and a midline laparotomy was performed to expose the uterine horns. A DNA plasmid solution consisting of either pCAG-GFP (Addgene, catalog $\# 11150 ; 4.8 \mu \mathrm{g} / \mu \mathrm{l})$ or pCAG-GFP $(1-3 \mu \mathrm{g} / \mu \mathrm{l})+$ pCAG-Rheb $^{\text {CA }}$ (also known as pCAG-Rheb ${ }^{\mathrm{S} 16 \mathrm{H}}$; gift from T. Maehama and K. Hanada; Maehama et al., 2008, 1.5, 2.5, or $3.5 \mu \mathrm{g} / \mu \mathrm{l}$ ) together with $0.03 \%$ Fast Green dye (for visualizing injection) was injected (1-1.5 $\mu$ l) into the right lateral ventricle of each embryo using a glass pipette. Following injection, a 5 $\mathrm{mm}$ tweezer-type electrode was positioned on the embryo head and six $42 \mathrm{~V}, 50 \mathrm{~ms}$ pulses at $950 \mathrm{~ms}$ intervals were applied using a pulse generator (ECM830; BTX) to electroporate the plasmid constructs into neural progenitor cells. We preferentially targeted expression in layer $2 / 3$ (L2/3) in the medial prefrontal cortex ( $\mathrm{mPFC}$ ). The uterine horns were returned to the abdominal cavity and embryos were allowed to continue with their normal development. Pups were screened at postnatal day $0(\mathrm{P} 0)$ to verify successful electroporation via expression of GFP on a fluorescenceenabled stereomicroscope (SZX16; Olympus) before recruitment for video-EEG monitoring.

EEG head mount implantation. Prefabricated EEG head mounts (Pinnacle Technology, catalog \#8201-EEG) were implanted in 2- to 4-monthold mice. Mice were anesthetized with isoflurane and positioned on a stereotaxic frame using ear bars. A rostrocaudal midline incision was made in the skin to expose the skull surface. Four pilot holes (two bilateral holes $1 \mathrm{~mm}$ anterior to bregma and two bilateral holes $5 \mathrm{~mm}$ posterior to bregma, each $1.5 \mathrm{~mm}$ lateral to sagittal suture) were tapped through the skull to dura using a 23-gauge needle to accommodate the EEG head mount. The head mount was attached on top of the skull by threading four stainless steel screws (Pinnacle Technology, catalog \#8209) into the pilot holes. Silver conductive paint (Electron Microscopy Science) was applied around the screw threads to ensure a solid connection with the head mount. The entire implant was insulated using dental acrylic. Mice were allowed to recover in their home cages for at least 5 days before video-EEG monitoring.

Video-EEG monitoring and analysis. Mice were housed in individual recording chambers in a light-, temperature-, and humidity-controlled room during video-EEG monitoring. Mice were freely moving inside the chambers and had ad libitum access to food and water. Synchronous video-EEG recording was acquired using a three-channel EEG tethered system (Pinnacle Technology, catalog \#8200-K1-iSE3) and Sirenia Acquisition software (Pinnacle Technology). Mice were continuously recorded $24 \mathrm{~h}$ /day for 5-7 consecutive days. The average recording duration per animal was $120.5 \pm 29.6 \mathrm{~h}( \pm \mathrm{SD})$.

Seizure frequency and duration were analyzed using Sirenia Seizure Basic software (Pinnacle Technology). All analyses were performed blinded to experimental groups. The entire EEG traces were manually reviewed for the occurrence of seizures, defined as a sudden onset of high-amplitude activity with a characteristic pattern of progressive frequency and amplitude changes over the course of the event lasting $\geq 10 \mathrm{~s}$. Seizure onset was defined by high-amplitude firing $>2 \times$ baseline and seizure termination was defined by a return to baseline activity. Video data were inspected for behavioral correlates including myoclonic jerks, tonic-clonic activities, convulsions, and loss of postural control (rearing and falling) and were used as secondary verification of seizures. For each individual animal, the mean number of seizures per day was obtained by dividing the total number of seizures by total recording hours and multiplied by 24 . For each individual animal, mean seizure duration was obtained by measuring and averaging the duration of each seizure within that animal.

The average starting ages of EEG recording for each of the experimental groups were as follows: $12.2 \pm 0.93$ (control), $11.3 \pm 0.27$ (low Rheb ${ }^{\mathrm{CA}}$ ), $11.2 \pm 0.38$ (intermediate Rheb ${ }^{\mathrm{CA}}$ ), and $14.8 \pm 3.80$ (high $\mathrm{Rheb}^{\mathrm{CA}}$ ) weeks ( $\left.\pm \mathrm{SD}\right)$. Although the average age was higher in the high 
Rheb ${ }^{\mathrm{CA}}$ group (range 9.4-19 weeks) compared with the other Rheb ${ }^{\mathrm{CA}}$ groups ( $p=0.0058$; one-way ANOVA with Tukey's post hoc test), there was no significant correlation between seizures and age within this group for the given age range $(r=0.3756, p=0.2272$; Spearman's rank-order correlation, data not shown).

Brain slice preparation and immunofluorescence staining. After the end of video-EEG monitoring, mice were deeply anesthetized with sodium pentobarbital ( $85 \mathrm{mg} / \mathrm{kg}$, i.p.) and transcardially perfused with ice-cold PBS, pH 7.4, followed by ice-cold 4\% PFA diluted in PBS. Whole brains were dissected and postfixed in $4 \%$ PFA for $2 \mathrm{~h}$ and then cryoprotected in $30 \%$ sucrose diluted in PBS for $24-48 \mathrm{~h}$ at $4^{\circ} \mathrm{C}$ until brains sank.

Brains were serially cut into $50-\mu \mathrm{m}$-thick coronal sections using a freezing microtome and stored in PBS $+0.01 \%$ sodium azide. One series of sections was mounted on slides and coverslipped with mounting media (ProLong Diamond Antifade Mountant; Invitrogen) to evaluate neuronal soma size, placement, and count. Other series of sections were used for immunofluorescence staining to evaluate expression of phosphorylated ribosomal protein $\mathrm{S} 6$ ( $\mathrm{p}-\mathrm{S} 6$; mTORC1 marker) and ionized calcium-binding adaptor molecule 1 (IBA1; microglia marker). For staining, free-floating sections were washed in PBS $+0.1 \%$ Triton X-100 (PBS-T) for $2 \times 10 \mathrm{~min}$ and permeabilized in PBS $+0.3 \%$ Triton X-100 for $20 \mathrm{~min}$. Sections were then incubated in blocking buffer (5\% goat serum $+0.3 \%$ BSA $+0.3 \%$ Triton X-100 in PBS) for $1 \mathrm{~h}$ at room temperature followed by incubation in primary antibodies (anti-rabbit p-S6, S240/244, Cell Signaling Technology, catalog \#5364, 1:2000, or anti-rabbit IBA1, Wako, catalog \#019-19741, 1:1000, diluted in blocking buffer) overnight at $4^{\circ} \mathrm{C}$. The following day, sections were washed in PBS-T for $3 \times 10 \mathrm{~min}$ and incubated in secondary antibodies (goat anti-rabbit IgG-647 plus; Invitrogen, catalog \#A3273, 1:1000, diluted in blocking buffer) for $1 \mathrm{~h}$ at room temperature. Sections were then washed in PBS-T for $3 \times 10 \mathrm{~min}$, incubated in DAPI for $10 \mathrm{~min}$, and rinsed in PBS before being air-dried and coverslipped with mounting media (ProLong Diamond Antifade Mountant; Invitrogen).

Microscopy and image analysis. Fluorescence images were acquired using a confocal microscope (Fluoview FV1000; Olympus) with $10 \times$ and $20 \times$ objective lenses. All image analyses were done using ImageJ and were performed blinded to experimental groups. Cell counts were performed using the Cell Counter plugin for ImageJ. Representative images were prepared using ImageJ and Adobe Photoshop. All images meant for comparison were uniformly processed.

Cortical p-S6 staining intensity was quantified from single optical section images containing both the ipsilateral (electroporated) and contralateral cortices within the same plane of view. Two to three brain sections were analyzed per animal. For each section, the mean gray value was measured in three randomly selected, nonoverlapping $120 \mu \mathrm{m} \times$ $120 \mu \mathrm{m}$ region of interests (ROIs) surrounding the electroporated cells on the ipsilateral cortex. Three ROIs surrounding non-electroporated cells on the contralateral cortex were also measured as internal controls. ROIs on the contralateral cortex were chosen to match the position of the ROIs on the ipsilateral cortex. All ROIs were selected within $300 \mu \mathrm{m}$ from the pial surface. Cellular p-S6 staining intensity was quantified from single optical section images of either the ipsilateral or contralateral cortex. Two to three brain sections were analyzed per animal. For each section, the mean gray value was measured in all visible, nonoverlapping $\mathrm{GFP}^{+}$cells on the ipsilateral cortex (by tracing around the cells) and in position-matched cells on the contralateral cortex. Sixteen to 101 neurons were analyzed per animal. Data for both cortical and cellular p-S6 staining intensity are reported as a percentage of contralateral cortex to avoid confounding effects of variabilities in the quality of sectioning or staining and for directly evaluating $\mathrm{Rheb}^{\mathrm{CA}}$-induced $\mathrm{p}$-S6 changes.

Neuron size and placement were analyzed from maximum intensity projection images created from a 20 - $\mu$ m-thick $z$-stack of optical sections taken at $2 \mu \mathrm{m}$ increments of the ipsilateral cortex. Neuron size was quantified by tracing the soma and measuring the area of $\mathrm{GFP}^{+}$cells. A total of 21-60 neurons from two to three brain sections were analyzed per animal. The percentage of neurons in $\mathrm{L} 2 / 3$ was quantified by counting all $\mathrm{GFP}^{+}$cells within an $850 \mu \mathrm{m} \times 850 \mu \mathrm{m}$ ROI surrounding the ipsilateral cortex. Cells within $300 \mu \mathrm{m}$ from the pial surface were considered correctly located in L2/3, whereas cells outside that boundary were consid- ered misplaced. The distribution of neurons was quantified by dividing the cortex into 10 evenly spaced bins (bin size: $85 \mu \mathrm{m}$ width $\times 675 \mu \mathrm{m}$ height) parallel to the pial surface in coronal sections and counting the number of GFP ${ }^{+}$cells in each bin. Only cells within the gray matter of the cortex were quantified. One brain section was analyzed per animal for cell placement analyses. Data are shown as the percentage of total GFP ${ }^{+}$ cells.

To determine the number of electroporated cells per brain section, a rostrocaudal series of $10-17$ coronal sections at $300 \mu \mathrm{m}$ intervals were scanned on a fluorescence microscope for the section with the most apparent number $\mathrm{GFP}^{+}$cells for each animal. Single optical section images were then acquired on a confocal microscope for the selected brain sections and the number of $\mathrm{GFP}^{+}$cells in the cortex was determined.

Microglia density and size were analyzed from sum slice and maximum intensity projection images created from a 20 - $\mu$ m-thick $z$-stack of optical sections taken at $2 \mu \mathrm{m}$ increments, respectively. Microglia density was determined by counting all IBA1 ${ }^{+}$cells within a $500 \mu \mathrm{m} \times 500 \mu \mathrm{m}$ ROI surrounding each of the ipsilateral and contralateral cortices. Two brain sections were analyzed per animal. Microglia size was quantified by tracing the soma and measuring the area of all nonoverlapping IBA1 ${ }^{+}$ cells within a $200 \mu \mathrm{m} \times 200 \mu \mathrm{m}$ ROI surrounding the ipsilateral cortex. A total of $25-51$ cells from two to three brain sections were analyzed per animal.

Experimental design and statistical analysis. A total of 7 control, 8 low Rheb $^{\mathrm{CA}}, 7$ intermediate Rheb ${ }^{\mathrm{CA}}$, and 12 high $\mathrm{Rheb}^{\mathrm{CA}}$ mice were monitored with video-EEG recording. Animals were then euthanized for post hoc histology and immunohistochemical analyses. Tissue was available for 7 control, 8 low Rheb ${ }^{\mathrm{CA}}$, 6 intermediate Rheb ${ }^{\mathrm{CA}}$ mice, and 5 high Rheb ${ }^{\mathrm{CA}}$ mice. Only four high Rheb ${ }^{\mathrm{CA}}$ mice had complete data for seizure correlation studies. Tissue from the high $\mathrm{Rheb}^{\mathrm{CA}}$ group was not available for microglia analysis and is therefore not included. Multiple brain sections were evaluated per animal. The exact number of slices and cells evaluated per animal for each experiment are outlined above in the "Microscopy and image analysis" section. All statistical analyses were performed using Prism 7 software (GraphPad Software). Differences among groups were assessed by one-way ANOVA, two-way repeated-measures ANOVA (with Tukey's or Bonferroni's post hoc test), Pearson's productmoment correlation, Spearman's rank-order correlation, or MannWhitney $U$ test, as appropriate. Individual tests are specified in the results, figure legends, and Table 1 . Repeat measures within an animal were averaged to yield one sample replicate for statistical analysis. For all analyses, sample size $(n)$ refers to number of animals. The significance level was set at $p<0.05$. All data are presented as mean \pm SEM.

\section{Results}

We previously reported that focal expression of Rheb ${ }^{\mathrm{CA}}$ in developing neurons by in utero electroporation in mice results in mTORC1 hyperactivation and typical features of TSC and FCD, including neuronal hypertrophy, ectopic neuron placement, and epilepsy (Gong et al., 2015; Hsieh et al., 2016; Lin et al., 2016). Here, we extend this model to examine whether the levels of mTORC1 hyperactivity affect the severity of these phenotypes (Fig. 1A,B). To induce differential mTORC1 activity levels in neurons, we electroporated three varying concentrations of the Rheb ${ }^{\mathrm{CA}}$ plasmid, $1.5,2.5$, and $3.5 \mu \mathrm{g} / \mu \mathrm{l}$ (hereafter referred as low, intermediate, and high, respectively), into the mouse cortex. We performed electroporation at E15, which selectively introduces the plasmid into neural progenitor cells that generate pyramidal neurons destined to L2/3. Moreover, we preferentially targeted expression in the mPFC because $50-90 \%$ of the focal malformations are found in the frontal lobes of patients (Braffman et al., 1992; Houser and Gomez, 1992; Baron and Barkovich, 1999; Colombo et al., 2009; Kabat and Król, 2012). In all conditions, mice were co-electroporated with a GFP reporter plasmid to visualize targeted cells. As controls, littermates were electroporated with GFP plasmid only. 
Table 1. Summary of statistical tests

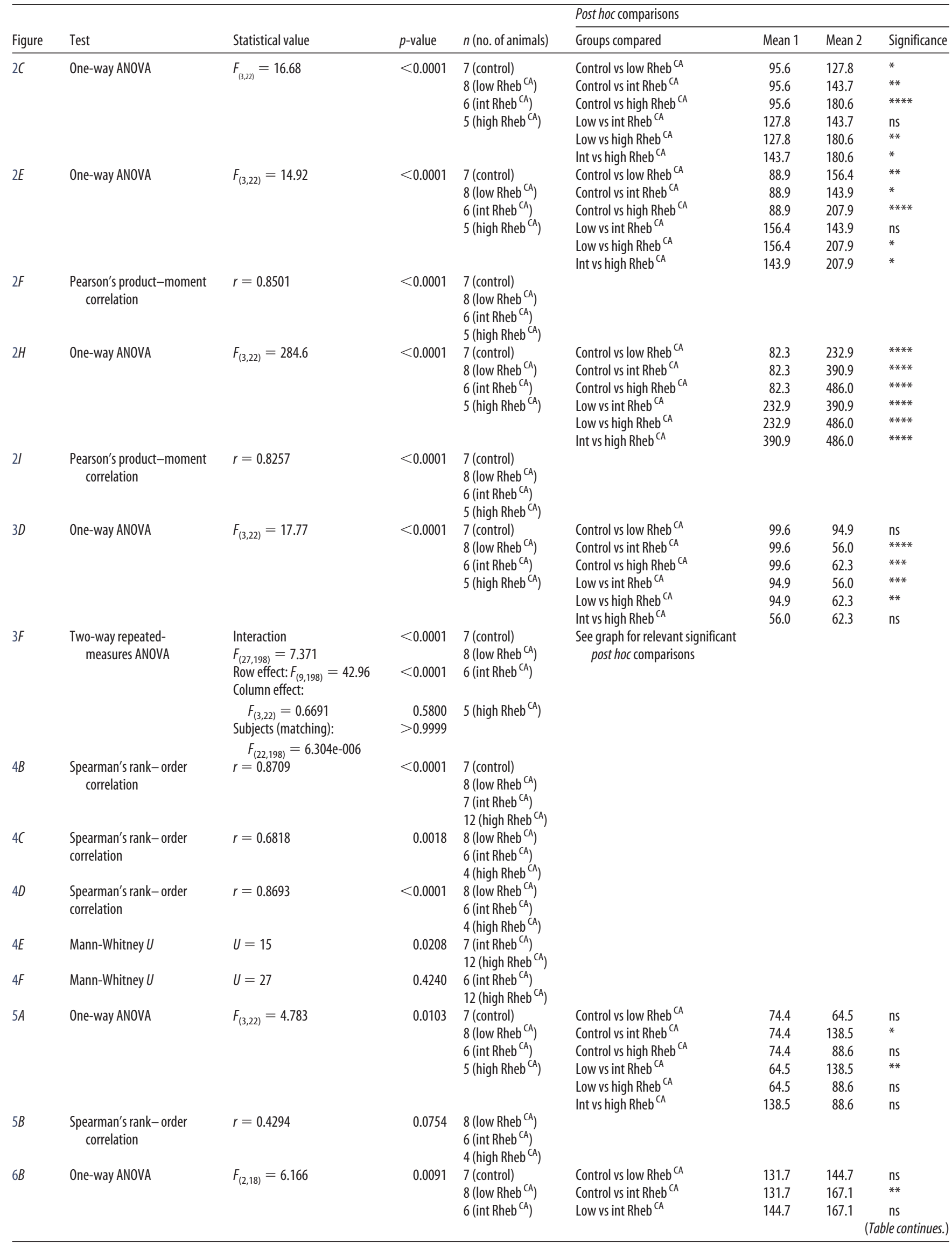


Table 1. Continued

\begin{tabular}{|c|c|c|c|c|c|c|c|c|}
\hline \multirow[b]{2}{*}{ Figure } & \multirow[b]{2}{*}{ Test } & \multirow[b]{2}{*}{ Statistical value } & \multirow[b]{2}{*}{$p$-value } & \multirow[b]{2}{*}{$n$ (no. of animals) } & \multicolumn{4}{|l|}{ Post hoc comparisons } \\
\hline & & & & & Groups compared & Mean 1 & Mean 2 & Significance \\
\hline $6 C$ & $\begin{array}{l}\text { Spearman's rank-order } \\
\text { correlation }\end{array}$ & $r=0.5502$ & 0.0441 & $\begin{array}{l}8 \text { (low Rheb cA) } \\
6 \text { (int Rheb }{ }^{C A} \text { ) }\end{array}$ & & & & \\
\hline \multirow[t]{11}{*}{$6 D$} & Two-way repeated- & Interaction: & 0.0414 & 7 (control) & Ipsi-contra & & & \\
\hline & measures ANOVA & $F_{(2,16)}=3.913$ & & 8 (low Rheb ${ }^{\mathrm{CA}}$ ) & Control & 131.7 & 136.0 & ns \\
\hline & & Row effect: & 0.0494 & 4 (int Rheb $\left.{ }^{C A}\right)^{\prime}$ & Low & 144.7 & 138.2 & ns \\
\hline & & $F_{(1,16)}=4.522$ & & & Intermediate & 179.3 & 163.5 & $*$ \\
\hline & & Column effect: & 0.0006 & & Ipsi & & & \\
\hline & & $F_{(2,16)}=12.05$ & & & Control vs low & 131.7 & 144.7 & ns \\
\hline & & Subjects (matching): & 0.0022 & & Control vs int & 131.7 & 179.3 & $* * * *$ \\
\hline & & $F_{(16,16)}=4.522$ & & & $\begin{array}{l}\text { Low vs int } \\
\text { Contra }\end{array}$ & 144.7 & 179.3 & $* * *$ \\
\hline & & & & & Control vs low & 136.0 & 138.2 & ns \\
\hline & & & & & Control vs int & 136.0 & 163.5 & $* *$ \\
\hline & & & & & Low vs int & 138.2 & 163.5 & * \\
\hline \multirow[t]{3}{*}{$6 E$} & One-way ANOVA & $F_{(2,18)}=7.472$ & 0.0043 & 7 (control) & Control vs low Rheb ${ }^{\text {CA }}$ & 49.7 & 51.6 & ns \\
\hline & & & & 8 (low Rheb ${ }^{\text {CA }}$ ) & Control vs int Rheb ${ }^{\mathrm{CA}}$ & 49.7 & 72.0 & $* *$ \\
\hline & & & & 6 (int Rheb ${ }^{\mathrm{CA}}$ ) & Low vs int Rheb ${ }^{C A}$ & 51.6 & 72.0 & * \\
\hline
\end{tabular}

${ }^{*} p<0.05,{ }^{* *} p<0.01,{ }^{* * *} p<0.001,{ }^{* * * *} p<0.0001$.

\section{Rheb $^{\mathrm{CA}}$ expression induces mTORC1 hyperactivation and neuronal hypertrophy in a dose-dependent manner}

To verify that increasing Rheb ${ }^{\mathrm{CA}}$ plasmid concentration leads to increased levels of mTORC1 hyperactivation, we performed immunofluorescence staining for p-S6, a common marker of mTORC1 activity (Ma and Blenis, 2009), in brain sections from 2- to 4-month-old control- and Rheb ${ }^{\mathrm{CA}}$-electroporated mice (Fig. 2A). Because seizure activity may lead to secondary increases in p-S6 throughout the brain (Buckmaster et al., 2009; Zeng et al., 2009), we assessed p-S6 staining intensity on the ipsilateral cortex and normalized to that of the non-electroporated contralateral side for each animal to directly evaluate Rheb ${ }^{\mathrm{CA}}$-induced $\mathrm{p}$-S6 changes. In addition, we assessed p-S6 intensity both at the levels of cortical tissue (Fig. 2B,C) and electroporated cells (Fig. 2D,E) to compare the two approaches. We found significant increases in both cortical and cellular p-S6 intensity in the low, intermediate, and high Rheb ${ }^{\mathrm{CA}}$ conditions compared with control. Moreover, the high Rheb ${ }^{\mathrm{CA}}$ condition displayed significantly increased cortical and cellular p-S6 intensity compared with both the low and intermediate Rheb ${ }^{\mathrm{CA}}$ conditions $(p<0.0001$; one-way ANOVA with Tukey's post hoc test; Fig. 2B-E, Table 1). Additional correlation analyses between cortical and cellular p-S6 intensity demonstrate a significant correlation between the two variables $(r=$ $0.8501, p<0.0001$; Pearson's product-moment correlation; Fig. $2 F$, Table 1), suggesting that both analysis approaches are valid. Together, these data support that increasing Rheb ${ }^{\mathrm{CA}}$ plasmid concentration leads to increased mTORC1 hyperactivity levels.

Because mTORC1 hyperactivation causes neuronal hypertrophy (Kwon et al., 2003; Tavazoie et al., 2005; Kassai et al., 2014), we also measured the soma size of $\mathrm{GFP}^{+}$electroporated neurons as a proxy for mTORC1 activity. We found significantly increased soma size of electroporated neurons in the low, intermediate, and high Rheb ${ }^{\mathrm{CA}}$ conditions compared with control. Moreover, the soma size of the intermediate Rheb ${ }^{\mathrm{CA}}$ neurons was significantly larger than those of the low Rheb ${ }^{\mathrm{CA}}$ neurons, whereas the soma size of the high Rheb ${ }^{\mathrm{CA}}$ neurons was significantly larger than both the low and intermediate Rheb ${ }^{\mathrm{CA}}$ neurons, indicating that increasing Rheb ${ }^{\mathrm{CA}}$ concentration leads to increased neuronal soma size ( $p<0.0001$; one-way ANOVA with Tukey's post hoc test; Fig. 2G,H, Table 1). Further evaluation of the relationship between neuronal soma size and p-S6 level revealed a significant positive correlation between these two markers of mTORC1 ac- tivity $(r=0.8257, p<0.0001$; Pearson's product moment-correlation; Fig. 2I, Table 1). Collectively, these data demonstrate that Rheb ${ }^{\mathrm{CA}}$ expression induces mTORC1 hyperactivation and neuronal hypertrophy in a dose-dependent manner in vivo.

\section{High but not low levels of mTORC1 hyperactivity cause ectopic neuronal placement}

We next evaluated the distribution of electroporated neurons in brain sections from 2- to 4-month-old control- and Rheb ${ }^{\mathrm{CA}}$ electroporated mice (Fig. $3 A$ ). As expected, all electroporated neurons in the control condition correctly integrated to L2/3. Similarly, most electroporated neurons in the low Rheb ${ }^{\mathrm{CA}}$ condition were properly positioned in L2/3, whereas only $\sim 50 \%$ of electroporated neurons in the intermediate and high Rheb ${ }^{\mathrm{CA}}$ conditions were found in L2/3. Quantification of the percentage of electroporated neurons in L2/3 revealed no statistical differences between the low Rheb ${ }^{\mathrm{CA}}$ and control conditions. In contrast, the intermediate and high Rheb ${ }^{\mathrm{CA}}$ conditions were significantly different from both the control and low Rheb ${ }^{\mathrm{CA}}$ conditions, but not from each other $(p<0.0001$; one-way ANOVA with Tukey's post hoc test; Fig. $3 B-D$, Table 1$)$. The binned distribution of electroporated neurons across the cortex was similar in control and low Rheb ${ }^{\mathrm{CA}}$ conditions but significantly different in the intermediate and high Rheb ${ }^{\mathrm{CA}}$ conditions relative to control ( $p<0.0001$, interaction; two-way repeated-measures ANOVA; Fig. 3E, F, Table 1). Together, these data indicate that higher but not lower levels of mTORC1 hyperactivation lead to ectopic neuronal placement. The finding that the low Rheb ${ }^{\mathrm{CA}}$ condition, which sufficiently induced neuronal hypertrophy (Fig. 2G,H), did not cause neuronal misplacement suggests that there is a different threshold level of mTORC1 hyperactivation required to induce neuronal misplacement compared with neuronal hypertrophy.

\section{Increasing levels of mTORC1 hyperactivity lead to increased seizure frequency}

To determine whether increasing levels of mTORC1 hyperactivity leads to a more severe seizure phenotype, we performed continuous video-EEG recording in 2- to 4-month-old control- and Rheb ${ }^{\mathrm{CA}}$-electroporated mice for 5-7 consecutive days. We detected no seizures in the control $(n=7 / 7)$ and low Rheb ${ }^{\mathrm{CA}}(n=$ $8 / 8)$ mice. In contrast, $86 \%$ of the intermediate Rheb ${ }^{\mathrm{CA}}$ mice 

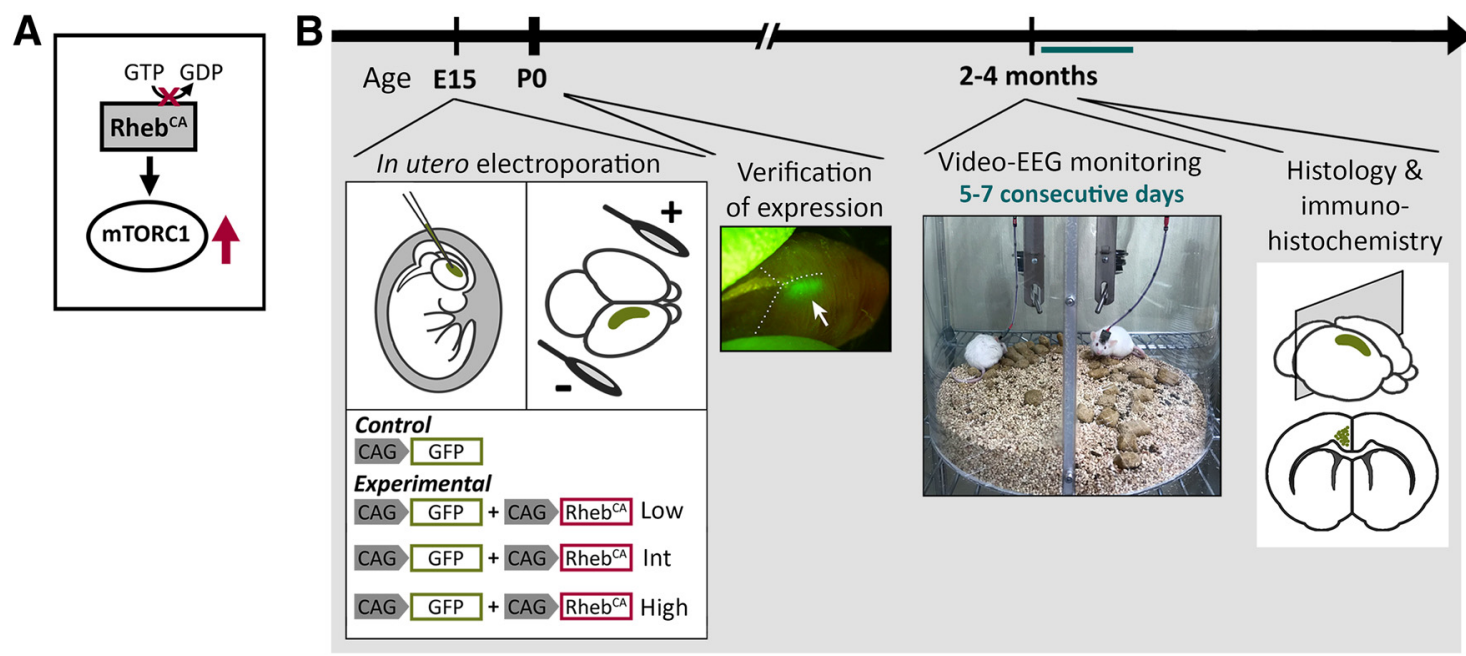

Figure 1. Experimental design and timeline. $A$, Simplified diagram of Rheb ${ }^{\text {CA }}$-induced mTORC1 activation. Rheb is a small GTPase upstream of mTORC1. GTP-bound Rheb directly binds to and activates mTORC1. The constitutively active mutant form of Rheb, Rheb ${ }^{\mathrm{CA}}$, selects for the GTP-bound form, thereby leading to persistent activation of mTORC1. B, Timeline for experiments. Mice were electroporated with GFP (control) or varying concentrations of GFP ${ }^{+}$Rheb $^{\text {CA }}$ plasmids at E15, targeting L2/3 neurons in the cortex. Pups were screened for successful electroporation via GFP expression at P0. Mice were monitored with video-EEG recording for $5-7$ consecutive days at $2-4$ months of age and afterward euthanized for histological and immunohistochemical analysis.

( $n=6 / 7)$ and $100 \%$ of the high Rheb ${ }^{\mathrm{CA}}$ mice $(n=12 / 12)$ exhibited spontaneous, recurrent seizures (Fig. 4A). Quantification of the daily seizure frequency revealed a significant positive correlation between Rheb ${ }^{\mathrm{CA}}$ plasmid concentration and seizure frequency $(r=0.8709, p<0.0001$; Spearman's rank-order correlation; Fig. 4B, Table 1). Further analyses revealed significant positive correlations between seizure frequency and p-S6 intensity $(r=0.6818, p<0.0018$; Spearman's rank-order correlation; Fig. $4 C$, Table 1), as well as between seizure frequency and neuronal soma size $(r=0.8693, p<0.0001$; Spearman's rankorder correlation; Fig. $4 D$, Table 1). The number of seizures per day was significantly increased in the high Rheb ${ }^{\mathrm{CA}}$ compared with the intermediate $\mathrm{Rheb}^{\mathrm{CA}}$ condition ( $p=0.0208$; MannWhitney $U$ test; Fig. $4 E$, Table 1 ). However, no statistical differences in the duration of seizures were observed between the two conditions ( $p=0.4240$; Mann-Whitney $U$ test; Fig. $4 F$, Table 1 ). The daily seizure frequency within individual animals remained overall consistent over the course of the recording period in both the intermediate and high Rheb ${ }^{\mathrm{CA}}$ conditions (Fig. 4G). Collectively, these findings support that increasing levels of mTORC1 hyperactivity leads to increased seizures in mice. The low Rheb ${ }^{\mathrm{CA}}$ condition did not result in seizures, in contrast to the intermediate and high Rheb ${ }^{\mathrm{CA}}$ conditions, suggesting that there is a threshold level of mTORC1 hyperactivation required for seizure induction. Furthermore, our data from the intermediate and high Rheb ${ }^{\mathrm{CA}}$ conditions support that, past the threshold level, there is a graded event in which increased mTORC1 hyperactivity levels leads to increased seizure frequency.

Given that the proportion of cells with aberrant mTORC1 activation (i.e., number of electroporated cells) may affect seizure severity, we compared the number of $\mathrm{GFP}^{+}$cells in the brain section with the highest $\mathrm{GFP}^{+}$cell count from a rostrocaudal series of sections for each animal. We found that the intermediate Rheb ${ }^{\text {CA }}$ condition had a higher $\mathrm{GFP}^{+}$cell count than the control and low Rheb ${ }^{\mathrm{CA}}$ conditions, whereas none of the other groups was different from each other $(p=0.0103$; one-way ANOVA with Tukey's post hoc test; Fig. 5A, Table 1). Nonetheless, there was no significant correlation between the number of $\mathrm{GFP}^{+}$cells and seizure frequency among the Rheb ${ }^{\mathrm{CA}}$ conditions $(r=0.4294, p=$ 0.0754; Spearman's rank-order correlation; Fig. $5 B$, Table 1), sup- porting that the observed intergroup differences in seizure frequency are due to differences in cellular mTORC1 hyperactivity levels rather than differences in the proportion of electroporated cells.

\section{Rheb ${ }^{\mathrm{CA}}$-induced seizures are associated with increased microglial activation}

Microglia activation, including alterations in microglia density and morphology, have been described in human TSC tubers and FCDII brain specimens (Boer et al., 2006, 2008a,b; Choi et al., 2009; Iyer et al., 2010), but it is unclear whether these microglia changes occur in the present mouse model. We thus immunostained for the microglia-specific protein IBA1 in brain sections from 2- to 4-month-old control, low Rheb ${ }^{\mathrm{CA}}$, and intermediate $\mathrm{Rheb}^{\mathrm{CA}}$ mice. Tissue was not available for the high Rheb ${ }^{\mathrm{CA}}$ condition and was therefore not included. We observed prominent increases in $\mathrm{IBA}^{+}$microglia staining in the intermediate Rhe$\mathrm{b}^{\mathrm{CA}}$ condition compared with the control and low Rheb ${ }^{\mathrm{CA}}$ conditions (Fig. 6A). Quantification of $\mathrm{IBA}^{+}$cell density in the ipsilateral cortex revealed a significant increase in the intermediate Rheb ${ }^{\mathrm{CA}}$ condition compared with control ( $p=0.0091$; oneway ANOVA with Tukey's post hoc test; Fig. 6B, Table 1), suggesting the presence of microglia activation. While analyzing tissue from the intermediate Rheb ${ }^{\mathrm{CA}}$ condition, we noticed a large intragroup difference in $\mathrm{IBA} 1^{+}$staining pattern, with some animals displaying marked increases in microglia density and others appearing similar to the control (open circles in Fig. $6 B$ ). To determine whether these changes could be associated with seizure activity, we performed correlation analyses between seizure frequency and $\mathrm{IBA}^{+}{ }^{+}$cell density. Indeed, we found a significant correlation between IBA1 ${ }^{+}$cell density and seizures $(r=$ 0.5502; $p=0.0441$; Spearman's rank-order correlation; Fig. $6 C$, Table 1 ). To further investigate whether increased $\mathrm{IBA}^{+}{ }^{+}$cell density reflects a global change induced by seizures or a localized effect associated with the electroporated, "disordered" neurons, we compared $\mathrm{IBA}^{+}{ }^{+}$cell density in the ipsilateral and contralateral cortices. The two animals from the intermediate Rheb ${ }^{\mathrm{CA}}$ conditions with $\leq 1$ seizure in 7 days were excluded from this analysis. We found that $\mathrm{IBA}^{+}{ }^{+}$cell density on both the ipsilateral and contralateral sides of the intermediate $\mathrm{Rheb}{ }^{\mathrm{CA}}$ condition was higher compared with the corresponding cortices in the control 
A

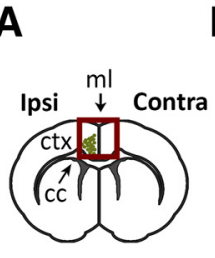

B

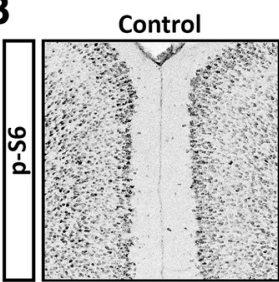

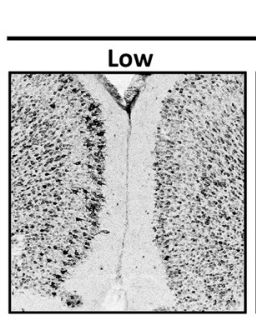

Rheb ${ }^{\text {CA }}$

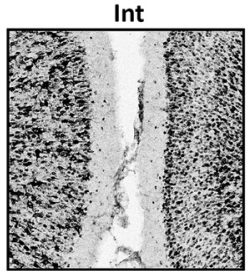

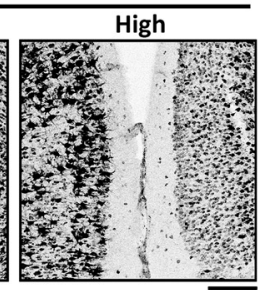

C

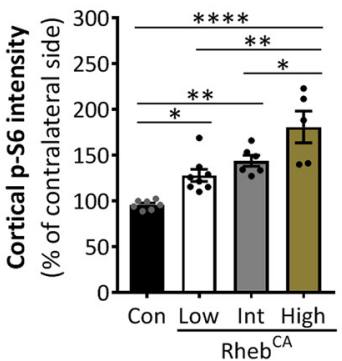

F

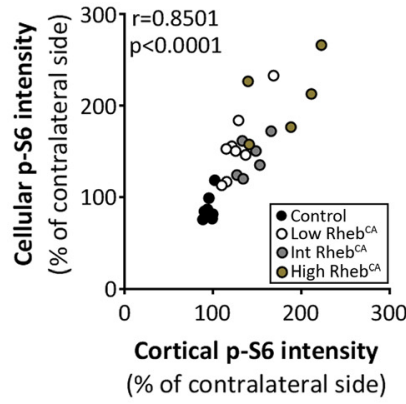

I

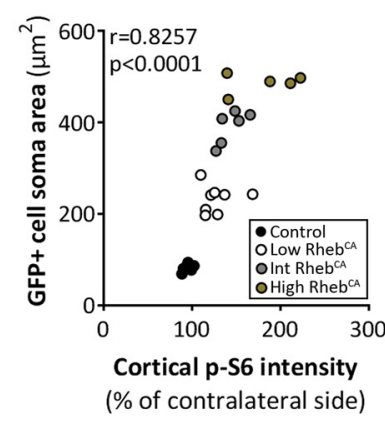

G

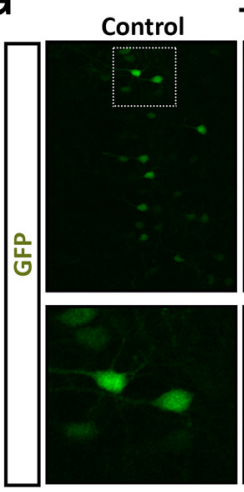

Rheb $^{\text {CA }}$

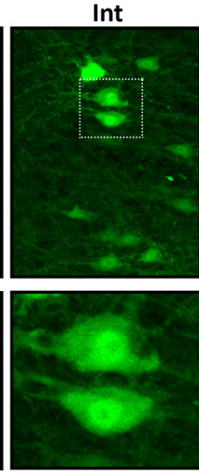

\section{Low}

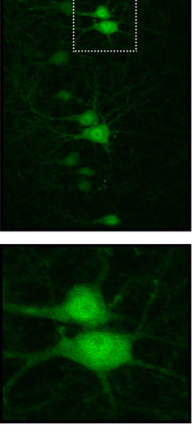

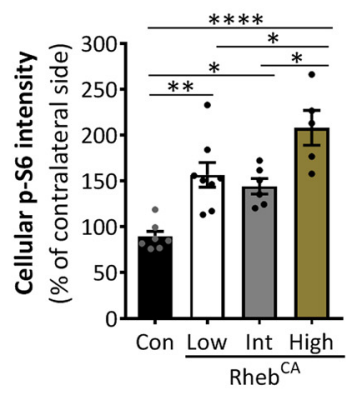

H
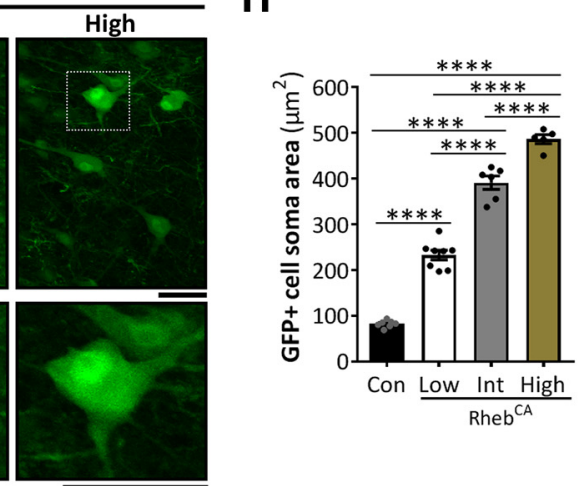

High

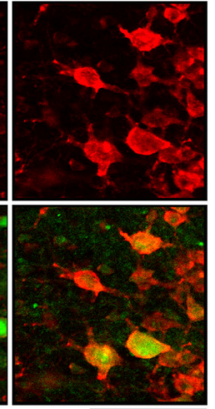

Figure 2. Rheb ${ }^{C A}$ expression induces $S 6$ phosphorylation and somatic hypertrophy in a dose-dependent manner. $A$, Diagram of a coronal brain section illustrating the electroporated region (green) of the cortex. Ipsilateral side is the electroporated cortex; contralateral side is the non-electroporated cortex. Red square indicates the area imaged in Figure $2 B$. Ipsi, Ipsilateral; Contra, contralateral; ctx, cortex; $c c$, corpus callosum; $\mathrm{ml}$, midline. $\boldsymbol{B}$, Inverted grayscale images of $\mathrm{p}$ - $\mathbf{S} 6$ staining in ipsilateral and contralateral cortices from control and low, intermediate, and high Rheb ${ }^{C A}$ mice. Scale bar, $200 \mu \mathrm{m}$. C, Quantification of $p$-S6 staining intensity in ipsilateral cortices. $p<0.0001$ by one-way AN0VA. Tukey's post hoc test: ${ }^{*} p<0.05,{ }^{* *} p<0.01,{ }^{* * * *} p<0.0001 . D$, Images of p-S6 staining (red) and GFP ${ }^{+}$cells (green) in ipsilateral cortices from control and low, intermediate, and high Rheb ${ }^{\text {CA }}$ mice. Scale bar, $100 \mu m$. E, Quantification of p-S6 staining intensity in GFP ${ }^{+}$ (electroporated) cells. $p<0.0001$ by one-way ANOVA. Tukey's post hoc test: ${ }^{*} p<0.05,{ }^{* *} p<0.01,{ }^{* * * *} p<0.0001$. F, Scatterplot of cellular versus cortical $p$-S6 staining intensity. $r=0.8501$, $p<0.0001$ by Pearson's product-moment correlation. G, Images of GFP ${ }^{+}$cells from control and low, intermediate, and high Rheb ${ }^{\mathrm{CA}}$ mice. White squares on the top panel are enlarged on the bottom panel. Scale bar, $50 \mu \mathrm{m}$ (top and bottom). $\boldsymbol{H}$, Quantification of GFP ${ }^{+}$cell soma size. $p<0.0001$ by one-way ANOVA. Tukey's post hoc test: ${ }^{* * * *} p<0.0001 . I$, Scatterplot of GFP ${ }^{+}$cell soma size versus cortical $p$-S6 staining intensity. $r=0.8257, p<0.0001$ by Pearson's product-moment correlation. Error bars indicate \pm SEM. Con, Control; Int, intermediate.

and low Rheb ${ }^{\mathrm{CA}}$ conditions $(p=0.0414$, interaction; two-way repeated-measures ANOVA with Bonferroni post hoc test; Fig. $6 D$, Table 1), suggesting that microglial activation are globally induced by seizures in this model. Interestingly, we also found a significant difference in $\mathrm{IBA}_{1}{ }^{+}$cell density between the ipsilateral and contralateral cortices within the intermediate Rheb ${ }^{\mathrm{CA}}$ condition, but not in the control and low Rheb ${ }^{\mathrm{CA}}$ conditions, suggesting that the presence of disordered neurons leads to a larger microglia activation.

Activated microglia undergo morphological changes from small cell bodies with ramified (branched) processes to enlarged cell bodies with long or short, thickened processes or ameboidshaped cells with no processes (Kettenmann et al., 2011). Quantification of $\mathrm{IBA}^{+}$cell size in the ipsilateral cortex revealed significantly larger cells in the intermediate Rheb ${ }^{\mathrm{CA}}$ condition compared with the control and low Rheb ${ }^{\mathrm{CA}}$ conditions, whereas no differences were observed between the control and low Rhe-
$\mathrm{b}^{\mathrm{CA}}$ conditions ( $p=0.0043$; one-way ANOVA with Tukey's post hoc test; Fig. 6E, Table 1). Further inspection of IBA1-stained tissue from control, low Rheb ${ }^{\mathrm{CA}}$, and intermediate Rheb ${ }^{\mathrm{CA}}$ mice revealed evident differences in the microglia morphological phenotypes between these groups. Although most microglia had the ramified phenotype in the control and low Rheb ${ }^{\mathrm{CA}}$ conditions, we identified at least four different types of microglia morphologies, including ramified, hypertrophic, bushy, and rod, that are consistent with activated microglia states in the intermediate Rheb ${ }^{\mathrm{CA}}$ condition (Fig. $6 F$ ). Overall, these findings further validate the presence of microglial activation in association with $\mathrm{Rheb}^{\mathrm{CA}}$-induced cortical malformations and seizures in this model.

\section{Discussion}

TSC and FCD result from a spectrum of pathogenic variants in the mTOR pathway that leads to differential mTOR hyperactivation (Crino, 2015). In this study, we demonstrate that the levels of 
A

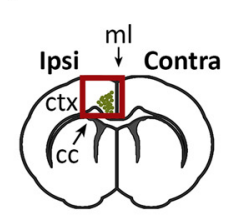

B

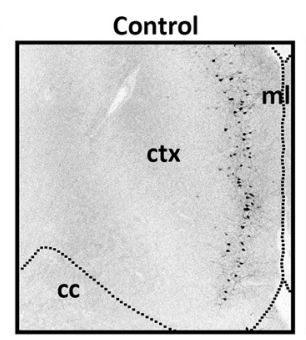

C

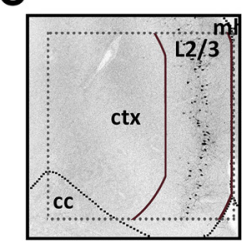

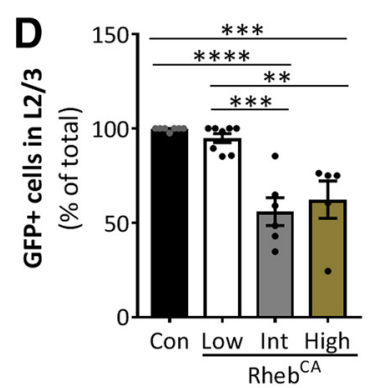

Rheb ${ }^{\text {CA }}$

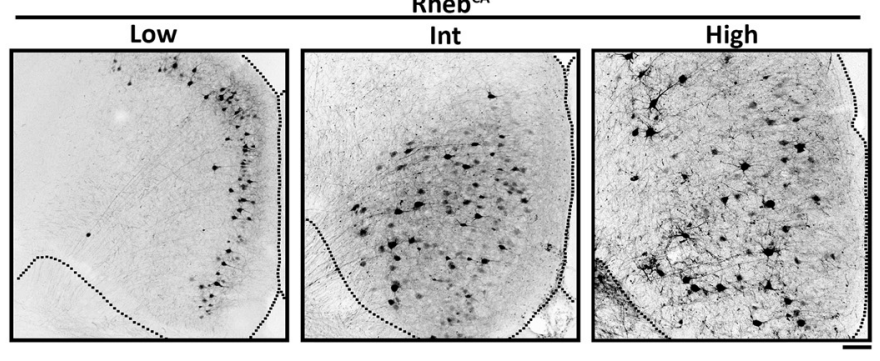

E

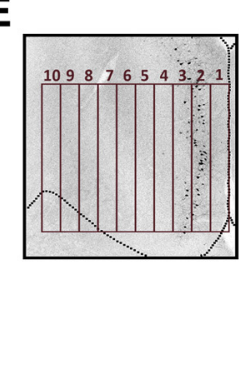

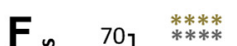

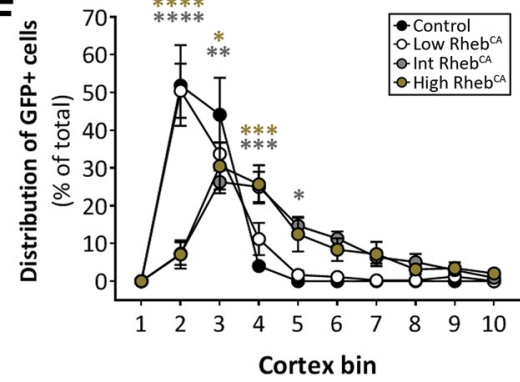

Figure 3. High but not low levels of mTORC1 hyperactivity cause ectopic neuronal placement. $A$, Diagram of a coronal brain section illustrating the electroporated region (green) of the cortex. Red square indicates the area imaged in Figure $3 B$. Ipsi, Ipsilateral; Contra, contralateral; ctx, cortex; cc, corpus callosum; ml, midline. $\boldsymbol{B}$, Inverted grayscale images of GFP ${ }^{+}$neurons in ipsilateral cortices from control and low, intermediate, and high Rheb ${ }^{\text {CA }}$ mice. Scale bar, $100 \mu \mathrm{m}$. C, Diagram of the ROI used to quantify the percentage of GFP ${ }^{+}$cells in L2/3. D, Quantification of the percentage of $\mathrm{GFP}^{+}$cells in L2/3.p $<0.0001$ by one-way ANOVA. Tukey's post hoc test: ${ }^{* *} p<0.01,{ }^{* * *} p<0.001,{ }^{* * * *} p<0.0001$. E, Diagram of the binning system used to quantify GFP ${ }^{+}$cell distribution in the cortex. $\boldsymbol{F}$, Quantification of GFP ${ }^{+}$cell distribution in the cortex. The number of GFP ${ }^{+}$cells in each bin (1-10) was divided by the total number of GFP ${ }^{+}$cells. $p<0.0001$ by tw0-way repeated-measures ANOVA. Tukey's post hoc test (simple main effects within cortex bins compared with control): ${ }^{*} p<0.01,{ }^{* *} p<0.01,{ }^{* * *} p<0.001,{ }^{* * * *} p<0.0001$. Error bars indicate \pm SEM. Con, Control; Int, intermediate.

mTOR hyperactivity correlate with the degree of neuronal hypertrophy, misplacement, and seizure frequency in an experimental TSC and FCD model. In addition, we describe the presence of microglia activation following mTOR-induced seizures.

The mTOR pathway regulates key processes involved in cell growth, including protein synthesis, energy metabolism, and autophagy, and aberrant mTOR activation has been implicated in epilepsy (Meng et al., 2013; Ostendorf and Wong, 2015; Saxton and Sabatini, 2017). Although the specific mechanisms underlying mTOR-induced epilepsy remain unclear, it is thought that structural changes following mTOR hyperactivation lead to circuit disorganization and altered intrinsic and network excitability that promote seizure generation (Lasarge and Danzer, 2014). Traditional methods for studying mTOR functions in epilepsy involve knocking out negative regulators of $\mathrm{mTOR}$ in transgenic mouse models to enhance mTOR activation (Feliciano et al., 2013; Ostendorf and Wong, 2015). Although these approaches have been instrumental in establishing the causative roles of mTOR signaling in epilepsy, investigating how the levels of mTOR hyperactivity influence epilepsy severity in these models have been limited to either the homozygosity or heterozygosity of the gene knock-out. In the present model, we expressed a constitutive activator of $\mathrm{mTORC} 1$, Rheb ${ }^{\mathrm{CA}}$, via in utero electroporation. This method allowed us to manipulate the levels of mTORC1 activity by varying the amount of electroporated Rhe$\mathrm{b}^{\mathrm{CA}}$ plasmids. Although we did not quantify the copy numbers that are transfected into cells under the different conditions, we speculate that higher copy numbers will be transfected with higher concentrations, thereby leading to higher levels of mTORC1 activation. Our data showing corresponding increases in p-S6 and neuronal soma size with increasing Rheb ${ }^{\mathrm{CA}}$ plasmid concentration support this notion. By varying the degree of mTORC1 hyperactivity, we show that there is a threshold level of mTORC1 hyperactivation required to induce seizures, followed by a graded event whereby increasing mTORC1 hyperactivity levels leads to increased seizure frequency. Our findings that higher mTOR hyperactivity levels contribute to more severe epilepsy suggest that differential levels of mTOR hyperactivation caused by distinct pathogenic variants may contribute to the variable phenotype severities in TSC and FCD.

Therapeutic strategies based on mTOR pathway inhibition for TSC- and FCD-related epilepsy are emerging. Although clinical trials with the mTOR inhibitor everolimus improved seizure control in many TSC patients, $30-40 \%$ of patients did not satisfactorily respond to treatment (Krueger et al., 2013; Wiegand et al., 2013; French et al., 2016). The wide array of variants along the mTOR pathway in TSC and FCD has raised the question of whether everolimus and other mTOR inhibitors provide the same efficacy for TSC or FCD resulting from distinct mTOR pathway variants (Iffland and Crino, 2017). Our findings that the levels of mTOR hyperactivity influence epilepsy severity may illuminate why current therapeutics based on mTOR inhibitors have variable outcomes on patients and suggest the need to consider patient gene variants in the context of mTOR activation levels in clinical assessments. As advanced genetic tools become more common, new pathogenic variants in the mTOR pathway are likely to be identified. Functional evaluation of these variants to determine their impact on the mTOR pathway and disease outcomes may aid in the establishment of a personalized and potentially more effective treatment regimen.

mTOR hyperactivation in the developing cortex causes neuronal hypertrophy and abnormal neuronal migration leading to cortical dyslamination (Feliciano et al., 2011; Kassai et al., 2014; Tsai et al., 2014; Hsieh et al., 2016; Lin et al., 2016). We demonstrate that neuronal soma size strongly correlates with the levels of mTOR hyperactivity and is a reliable readout of cellular mTOR activity level. Our cell size data showed a sigmoidal increase with increasing Rheb ${ }^{\mathrm{CA}}$ concentrations, suggesting that there is a pla- 
A
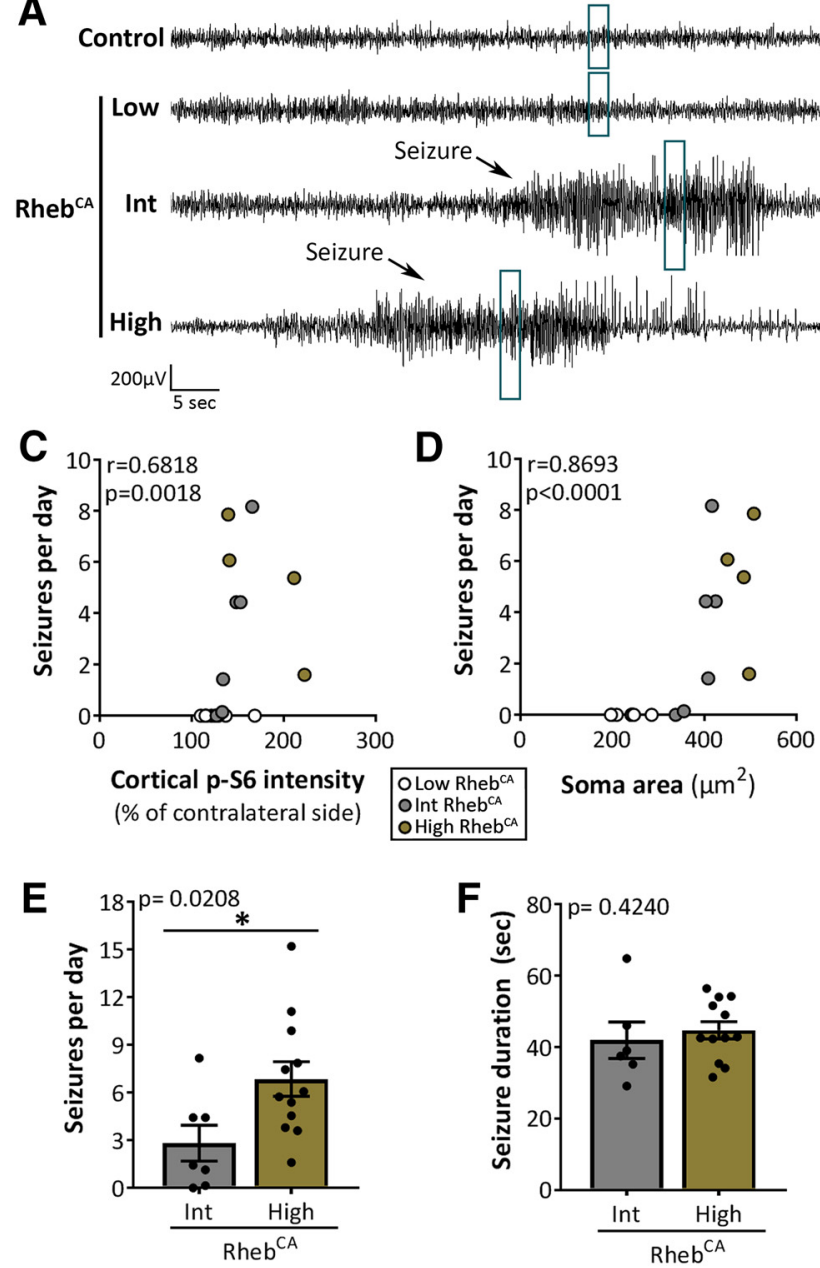

F

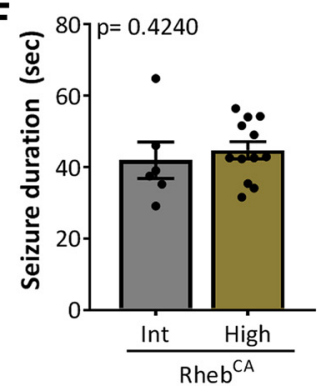

B

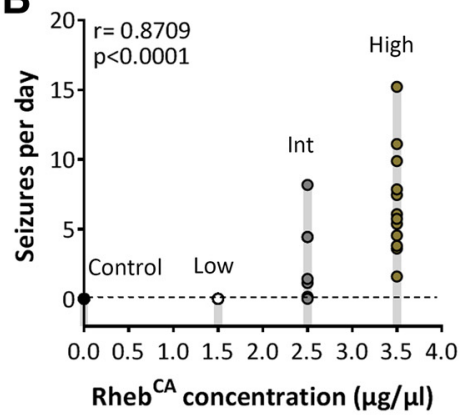

G

$200 \mu \mathrm{V} \frac{\mathrm{L}}{0.5 \mathrm{sec}}$

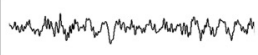

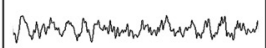

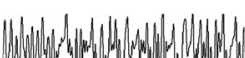

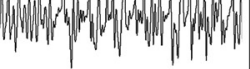

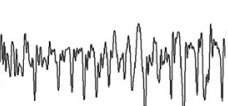

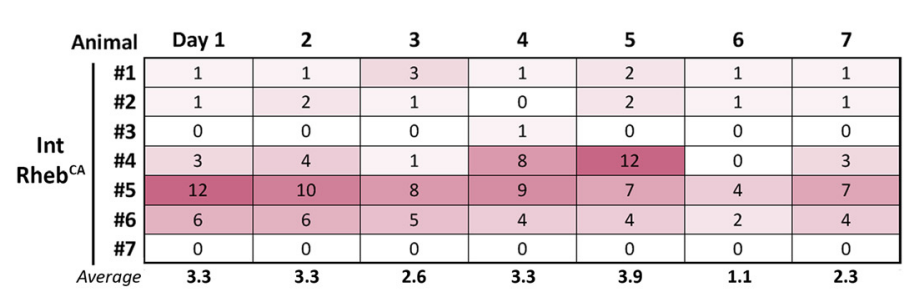

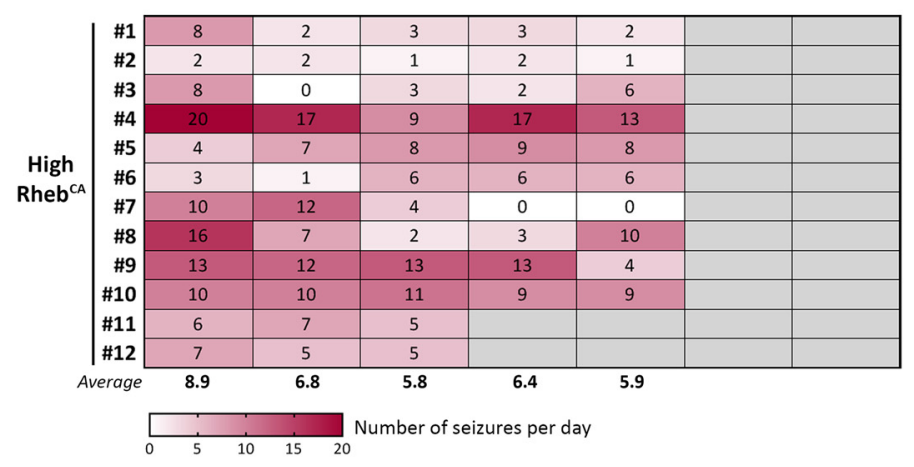

Figure 4. Increasing levels of mTORC1 hyperactivity lead to increased seizure frequency. $\boldsymbol{A}$, Representative EEG traces from control and low, intermediate, and high Rheb ${ }^{\mathrm{CA}}$ mice. Blue rectangles denote traces enlarged on the right. $\boldsymbol{B}$, Correlation between Rheb ${ }^{C A}$ plasmid concentration and mean number of seizures per day. $r=0.8709, p<0.0001$ by Spearman's rank- order correlation. C, Scatterplot of mean number of seizures per day versus cortical $p$-S6 intensity. $r=0.6818, p=0.0018$ by Spearman's rank- order correlation. $D$, Scatterplot of mean number of seizures per day versus GFP ${ }^{+}$cell soma size. $r=0.8693, p<0.0001$ by Spearman's rank- order correlation. $\boldsymbol{E}$, Mean number of seizures per day. ${ }^{*} p=0.0208$ by Mann-Whitney $U$ test. $\boldsymbol{F}$, Mean seizure duration. One of the intermediate Rheb ${ }^{\mathrm{CA}}$ mice did not exhibit seizures and was therefore not included in this analysis. $p=0.4240$ by Mann-Whitney $U$ test. $G$, Heat map showing the number of seizures per day over the total recording period (5-7 days) for individual animals. Error bars indicate \pm SEM. Int, Intermediate.

teau level for either mTOR activation or cell size. Nonetheless, determining the plateau level may be technically challenging as excess plasmid concentrations could be toxic to cells. Additionally, we show that high but not low levels of mTORC1 hyperactivation result in neuronal misplacement. We found most neurons in the low Rheb ${ }^{\mathrm{CA}}$ condition to be in the correct cortical layers despite having mTORC1 hyperactivation and neuronal hypertrophy. This finding suggests that there is a threshold level of mTORC1 hyperactivity needed for neuronal misplacement that is different than for neuronal hypertrophy.

Until recently, the mechanisms by which mTOR hyperactivation induce cortical dyslamination has been unknown. A
A

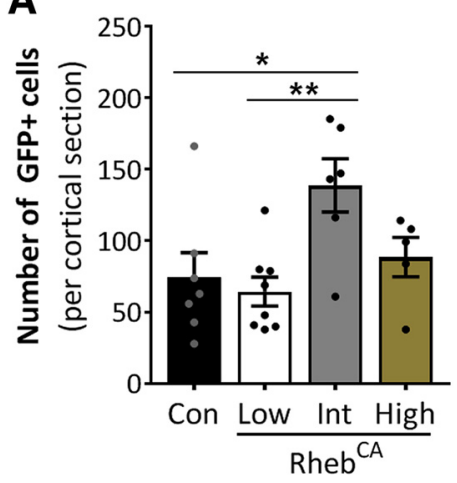

B

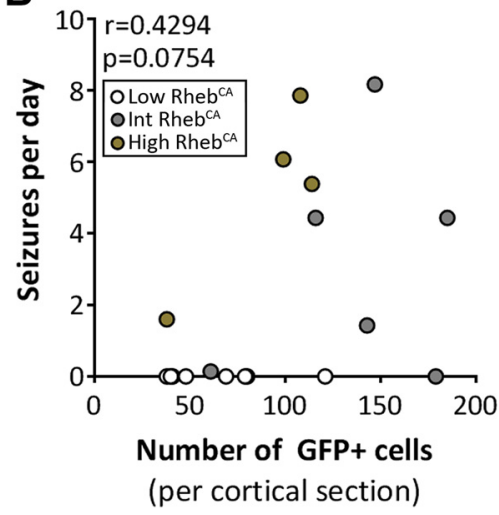

Figure 5. The number of electroporated cells does not affect intergroup differences in seizure frequency. $\boldsymbol{A}$, Quantification of the number of $\mathrm{GFP}^{+}$cells in single cortical sections containing the highest GFP ${ }^{+}$cell count from control and low, intermediate, and high Rheb ${ }^{\text {CA }}$ mice. $p=0.0103$ by one-way ANOVA. Tukey's post hoc test: ${ }^{*} p<0.05,{ }^{* *} p<0.01$. B, Scatterplot of mean number of seizures per day versus number of GFP ${ }^{+}$cells per single cortical section. $r=0.4294, p=0.0754$ by Spearman's rank- order correlation. Error bars indicate \pm SEM. Con, Control; Int, intermediate. 
A

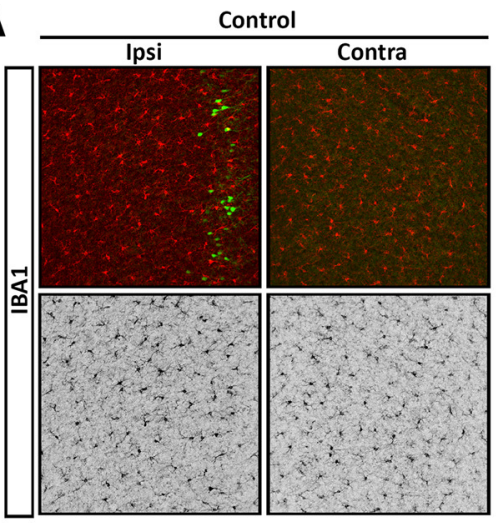

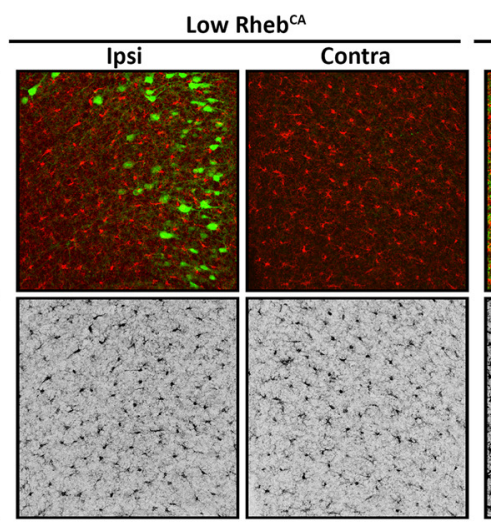

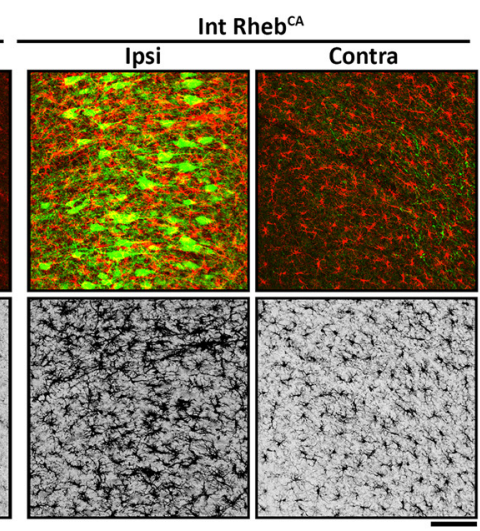

B
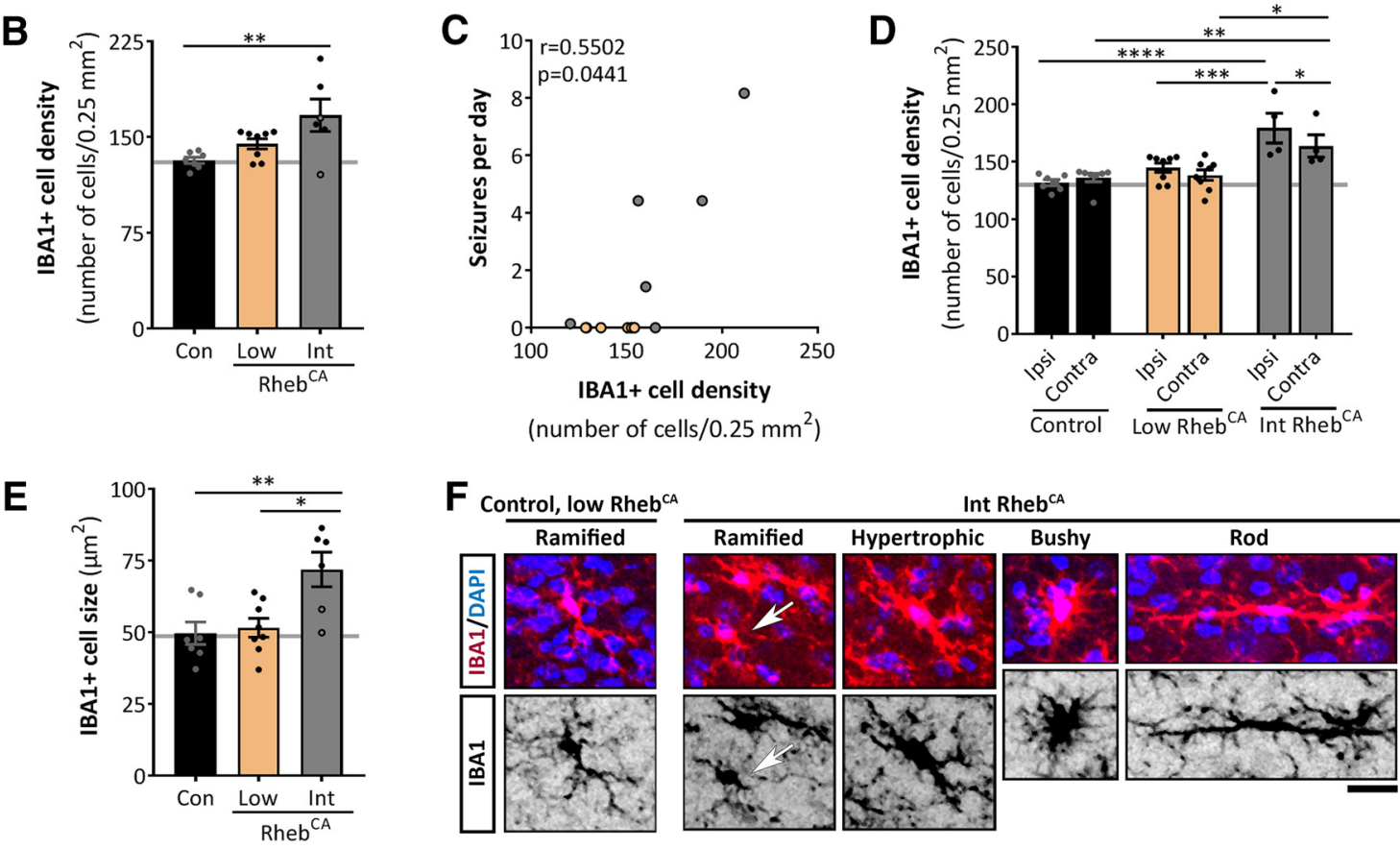

Figure 6. Rheb ${ }^{\mathrm{CA}}$-induced seizures are associated with increased microglial activation. $\boldsymbol{A}$, Images of IBA1 staining in ipsilateral and contralateral cortices from control, low Rheb ${ }^{\mathrm{CA}}$, and intermediate Rheb ${ }^{\mathrm{CA}}$ mice. Top, IBA1 staining (red) and GFP ${ }^{+}$cells (green). Bottom, Inverted grayscale images of the same IBA1 staining as in the top panels. Scale bar, $100 \mu \mathrm{m}$. Ipsi, Ipsilateral; Contra, contralateral. $\boldsymbol{B}$, Quantification of IBA $1^{+}$cell density in ipsilateral cortices. Open circles within the intermediate Rheb ${ }^{\mathrm{CA}}$ condition indicate animals with $\leq 1$ seizure. $p=0.0091$ by one-way ANOVA. Tukey's post hoc test: ${ }^{* *} p<0.001$. C, Scatterplot of mean number of seizures per day versus IBA1 ${ }^{+}$cell density. $r=0.5502, p=0.0441$ by Spearman's rank- order correlation. $\boldsymbol{D}$, Quantification of IBA ${ }^{+}$cell density in ipsilateral and contralateral cortices. $p=0.0414$ by two-way repeated-measures ANOVA. Bonferroni post hoc test: ${ }^{*} p<0.01,{ }^{* *} p<0.01$, ${ }^{* * *} p<0.001$, ${ }^{* * * *} p<0.0001$.E, Quantification of IBA1 ${ }^{+}$cell size in ipsilateral cortices. Open circles within the intermediate Rheb ${ }^{C A}$ condition indicate animals with $\leq 1$ seizure. $p=0.0043$ by one-way ANOVA. Tukey's post hoc test: ${ }^{*} p<0.05,{ }^{* *} p<0.01$. $\boldsymbol{F}$, Representative images of IBA1-labeled microglia morphology in tissue from control, low Rheb ${ }^{\mathrm{CA}}$, and intermediate Rheb ${ }^{\mathrm{CA}}$ mice. Top, IBA1 staining (red) and DAPI-stained nuclei (blue). Bottom, Inverted grayscale images of the same IBA1 staining as in the top panels. The most apparent microglia morphology observed in control and low Rheb ${ }^{C A}$ tissue was ramified, whereas microglia in intermediate Rheb ${ }^{\mathrm{CA}}$ tissue displayed a variety of morphologies, including ramified (indicated by white arrow), hypertrophic, bushy, and rod. Error bars indicate \pm SEM. Con, Control; Int, intermediate.

new study by Park et al. (2018) reported that brain somatic mutations in the MTOR gene lead to neuronal misplacement and cortical dyslamination by disrupting autophagy-mediated neuronal ciliogenesis. Therefore, defective ciliogenesis is a potential mechanism by which mTOR hyperactivation induced neuronal misplacement in our model. Although neuronal misplacement seems to parallel seizure occurrence (i.e., no misplacement or seizures in low Rheb ${ }^{\mathrm{CA}}$, misplacement and seizures in intermediate and high $\mathrm{Rheb}^{\mathrm{CA}}$ ), the role of cortical dyslamination in epileptogenesis is unclear. We previously used an inducible Rheb ${ }^{\mathrm{CA}}$ plasmid to bypass neuronal misplacement and observed that misplacement is not required for seizures and neuronal hypertrophy (Hsieh et al., 2016). This finding was corroborated by Park et al. (2018). Nonetheless, the specific contribution of cor- tical dyslamination to seizure severity and other neurological manifestations associated with mTOR hyperactivation remain elusive and additional studies are needed to elucidate these issues.

Studies on brain somatic mutations along the mTOR pathway have reported that the percentage of cells carrying the mutations correlate with the extent of cortical malformations and phenotypic severity (Blümcke and Sarnat, 2016; D'Gama et al., 2017). Given that the proportion of cells with aberrant mTORC1 activation (i.e., number of electroporated cells) may affect epilepsy severity in our studies, we compared the number of GFP ${ }^{+}$cells in individual animals. We found no correlation between $\mathrm{GFP}^{+}$cell count and seizure frequency, suggesting the identified intergroup differences in seizures is due to differences in cellular mTOR hyperactivity levels rather than the proportion of cells with 
mTORC1 hyperactivation. Nonetheless, we do not preclude the possibility that, within groups of similar Rheb ${ }^{\mathrm{CA}}$ concentrations, increasing the number of electroporated cells or brain region could lead to worsening seizures. Interestingly, studies by Pun et al. (2012) using genetic knock-out of Pten, a negative regulator of mTOR, in mice showed that Pten loss in $9-24 \%$ of hippocampal cells sufficiently induced seizures, whereas $<1 \%$ Pten loss did not. Collectively, we speculate that both the degree of mTOR hyperactivity within cells, as shown here, and the number of affected cells contribute to epilepsy severity.

Activation of microglia, the main immune cells of the CNS, have been found in brain specimens from TSC and FCD patients (Boer et al., 2006, 2008a,b; Choi et al., 2009; Iyer et al., 2010). Our data in mice demonstrating changes in microglia density and morphology corroborate the findings in humans. Interestingly, we observed microglial changes only in animals that displayed recurrent seizures and in both the ipsilateral and contralateral cortices, suggesting that microglia activation occurs as a consequence of seizures. Whether this represents a neurotoxic or neuroprotective mechanism is unclear and constitutes an active field of research (Eyo et al., 2017; Hiragi et al., 2018).

In addition to the biological implications of our study, we present practical modifications to a previously described in utero electroporation-based mouse model that allows for the titration of seizure frequency and generation of a mild to severe epilepsy phenotype as applicable for preclinical drug testing and mechanistic studies (Hsieh et al., 2016). For example, electroporation using lower Rheb ${ }^{\mathrm{CA}}$ plasmid concentrations can be used to generate mice for examining cellular or behavioral effects of mTOR hyperactivation in the absence of secondary changes due to seizures, whereas higher Rheb ${ }^{\mathrm{CA}}$ concentrations can be used to generate mice with frequent seizures to evaluate potential antiepileptic treatments. We emphasize that the Rheb ${ }^{\mathrm{CA}}$ concentrations described in this study are relative and not absolute. Investigator-dependent variability in plasmid preparation, injection volume, and electroporation techniques as well as electroporation parameters (i.e., voltage, number of pulses, etc.) could yield variable results. Therefore, investigators using this model should empirically determine the optimal dose for their experiments. Nonetheless, we demonstrate that a graded increase in Rheb ${ }^{\text {CA }}$ plasmid concentration results in increased mTOR hyperactivity levels and more severe phenotypes.

In summary, we show here that mTOR hyperactivity levels influence the severity of epilepsy and associated neuropathology in an experimental model of TSC and FCD. Our study suggests that differences in mTOR hyperactivity levels may contribute to the broad range of phenotypic severity in TSC and FCD and support the need to evaluate mTOR hyperactivity levels resulting from distinct patient variants. Future studies to address whether increasing mTOR hyperactivity levels worsen other behavioral and cognitive phenotypes may provide additional insights into the role of mTOR signaling in these aspects of TSC and FCD. Finally, whereas we limited our study to TSC and FCD strictly based on pathological definitions, our findings are potentially broadly applicable to other mTORopathies.

\section{References}

Aronica E, Becker AJ, Spreafico R (2012) Malformations of cortical development. Brain Pathol 22:380-401.

Baron Y, Barkovich AJ (1999) MR imaging of tuberous sclerosis in neonates and young infants. AJNR Am J Neuroradiol 20:907-916.

Blümcke I, Spreafico R, Haaker G, Coras R, Kobow K, Bien CG, Pfäfflin M, Elger C, Widman G, Schramm J, Becker A, Braun KP, Leijten F, Baayen JC, Aronica E, Chassoux F, Hamer H, Stefan H, Rössler K, Thom M, et al.
(2017) Histopathological findings in brain tissue obtained during epilepsy surgery. N Engl J Med 377:1648-1656.

Blümcke I, Sarnat HB (2016) Somatic mutations rather than viral infection classify focal cortical dysplasia type II as mTORopathy. Curr Opin Neurol 29:388-395.

Boer K, Spliet WG, van Rijen PC, Redeker S, Troost D, Aronica E (2006) Evidence of activated microglia in focal cortical dysplasia. J Neuroimmunol 173:188-195.

Boer K, Troost D, Jansen F, Nellist M, van den Ouweland AM, Geurts JJ, Spliet WG, Crino P, Aronica E (2008a) Clinicopathological and immunohistochemical findings in an autopsy case of tuberous sclerosis complex. Neuropathology 28:577-590.

Boer K, Jansen F, Nellist M, Redeker S, van den Ouweland AM, Spliet WG, van Nieuwenhuizen O, Troost D, Crino PB, Aronica E (2008b) Inflammatory processes in cortical tubers and subependymal giant cell tumors of tuberous sclerosis complex. Epilepsy Res 78:7-21.

Braffman BH, Bilaniuk LT, Naidich TP, Altman NR, Post MJ, Quencer RM, Zimmerman RA, Brody BA (1992) MR imaging of tuberous sclerosis: pathogenesis of this phakomatosis, use of gadopentetate dimeglumine, and literature review. Radiology 183:227-238.

Buckmaster PS, Ingram EA, Wen X (2009) Inhibition of the mammalian target of rapamycin signaling pathway suppresses dentate granule cell axon sprouting in a rodent model of temporal lobe epilepsy. J Neurosci 29:8259-8269.

Choi J, Nordli DR Jr, Alden TD, DiPatri A Jr, Laux L, Kelley K, Rosenow J, Schuele SU, Rajaram V, Koh S (2009) Cellular injury and neuroinflammation in children with chronic intractable epilepsy. J Neuroinflammation 6:38.

Colombo N, Salamon N, Raybaud C, Ozkara C, Barkovich AJ (2009) Imaging of malformations of cortical development. Epileptic Disord 11:194205 .

Crino PB (2015) mTOR signaling in epilepsy: insights from malformations of cortical development. Cold Spring Harb Perspect Med 5:a022442.

Curatolo P, Moavero R, Roberto D, Graziola F (2015) Genotype/Phenotype correlations in tuberous sclerosis complex. Semin Pediatr Neurol 22:259_ 273.

D'Gama AM, Woodworth MB, Hossain AA, Bizzotto S, Hatem NE, LaCoursiere CM, Najm I, Ying Z, Yang E, Barkovich AJ, Kwiatkowski DJ, Vinters HV, Madsen JR, Mathern GW, Blümcke I, Poduri A, Walsh CA (2017) Somatic mutations activating the mTOR pathway in dorsal telencephalic progenitors cause a continuum of cortical dysplasias. Cell Rep 21:37543766.

Eyo UB, Murugan M, Wu LJ (2017) Microglia-neuron communication in epilepsy. Glia 65:5-18.

Feliciano DM, Su T, Lopez J, Platel JC, Bordey A (2011) Single-cell Tscl knockout during corticogenesis generates tuber-like lesions and reduces seizure threshold in mice. J Clin Invest 121:1596-1607.

Feliciano DM, Lin TV, Hartman NW, Bartley CM, Kubera C, Hsieh L, Lafourcade C, O'Keefe RA, Bordey A (2013) A circuitry and biochemical basis for tuberous sclerosis symptoms: from epilepsy to neurocognitive deficits. Int J Dev Neurosci 31:667-678.

French JA, Lawson JA, Yapici Z, Ikeda H, Polster T, Nabbout R, Curatolo P, de Vries PJ, Dlugos DJ, Berkowitz N, Voi M, Peyrard S, Pelov D, Franz DN (2016) Adjunctive everolimus therapy for treatment-resistant focalonset seizures associated with tuberous sclerosis (EXIST-3): a phase 3 , randomised, double-blind, placebo-controlled study. Lancet 388:21532163.

Gong X, Zhang L, Huang T, Lin TV, Miyares L, Wen J, Hsieh L, Bordey A (2015) Activating the translational repressor 4E-BP or reducing S6KGSK3beta activity prevents accelerated axon growth induced by hyperactive mTOR in vivo. Hum Mol Genet 24:5746-5758.

Grabiner BC, Nardi V, Birsoy K, Possemato R, Shen K, Sinha S, Jordan A, Beck AH, Sabatini DM (2014) A diverse array of cancer-associated MTOR mutations are hyperactivating and can predict rapamycin sensitivity. Cancer Discov 4:554-563.

Harvey AS, Cross JH, Shinnar S, Mathern GW; ILAE Pediatric Epilepsy Surgery Survey Taskforce (2008) Defining the spectrum of international practice in pediatric epilepsy surgery patients. Epilepsia 49:146-155.

Hiragi T, Ikegaya Y, Koyama R (2018) Microglia after seizures and in epilepsy. Cells 7:E26.

Houser OW, Gomez MR (1992) CT and MR imaging of intracranial tuberous sclerosis. J Dermatol 19:904-908. 
Hsieh LS, Wen JH, Claycomb K, Huang Y, Harrsch FA, Naegele JR, Hyder F, Buchanan GF, Bordey A (2016) Convulsive seizures from experimental focal cortical dysplasia occur independently of cell misplacement. Nat Commun 7:11753.

Iffland PH 2nd, Crino PB (2016) Sending mixed signals: the expanding role of molecular cascade mutations in malformations of cortical development and epilepsy. Epilepsy Curr 16:158-163.

Iffland PH 2nd, Crino PB (2017) Focal cortical dysplasia: gene mutations, cell signaling, and therapeutic implications. Annu Rev Pathol 12:547-571.

Iyer A, Zurolo E, Spliet WG, van Rijen PC, Baayen JC, Gorter JA, Aronica E (2010) Evaluation of the innate and adaptive immunity in type I and type II focal cortical dysplasias. Epilepsia 51:1763-1773.

Kabat J, Król P (2012) Focal cortical dysplasia: review. Pol J Radiol 77:35-43.

Kassai H, Sugaya Y, Noda S, Nakao K, Maeda T, Kano M, Aiba A (2014) Selective activation of mTORC1 signaling recapitulates microcephaly, tuberous sclerosis, and neurodegenerative diseases. Cell Rep 7:1626-1639.

Kettenmann H, Hanisch UK, Noda M, Verkhratsky A (2011) Physiology of microglia. Physiol Rev 91:461-553.

Krueger DA, Wilfong AA, Holland-Bouley K, Anderson AE, Agricola K, Tudor C, Mays M, Lopez CM, Kim MO, Franz DN (2013) Everolimus treatment of refractory epilepsy in tuberous sclerosis complex. Ann Neurol 74:679-687.

Kwon CH, Zhu X, Zhang J, Baker SJ (2003) mTor is required for hypertrophy of pten-deficient neuronal soma in vivo. Proc Natl Acad Sci U S A 100:12923-12928.

Lasarge CL, Danzer SC (2014) Mechanisms regulating neuronal excitability and seizure development following mTOR pathway hyperactivation. Front Mol Neurosci 7:18.

Leventer RJ, Guerrini R, Dobyns WB (2008) Malformations of cortical development and epilepsy. Dialogues Clin Neurosci 10:47-62.

Lim JS, Kim WI, Kang HC, Kim SH, Park AH, Park EK, Cho YW, Kim S, Kim HM, Kim JA, Kim J, Rhee H, Kang SG, Kim HD, Kim D, Kim DS, Lee JH (2015) Brain somatic mutations in MTOR cause focal cortical dysplasia type II leading to intractable epilepsy. Nat Med 21:395-400.

Lim JS, Gopalappa R, Kim SH, Ramakrishna S, Lee M, Kim WI, Kim J, Park SM, Lee J, Oh JH, Kim HD, Park CH, Lee JS, Kim S, Kim DS, Han JM, Kang HC, Kim HH, Lee JH (2017) Somatic mutations in TSC1 and TSC2 cause focal cortical dysplasia. Am J Hum Genet 100:454-472.

Lin TV, Hsieh L, Kimura T, Malone TJ, Bordey A (2016) Normalizing translation through $4 \mathrm{E}-\mathrm{BP}$ prevents mTOR-driven cortical mislamination and ameliorates aberrant neuron integration. Proc Natl Acad Sci U S A 113:11330-11335.

Lipton JO, Sahin M (2014) The neurology of mTOR. Neuron 84:275-291.

Ma XM, Blenis J (2009) Molecular mechanisms of mTOR-mediated translational control. Nat Rev Mol Cell Biol 10:307-318.

Maehama T, Tanaka M, Nishina H, Murakami M, Kanaho Y, Hanada K (2008) RalA functions as an indispensable signal mediator for the nutrient-sensing system. J Biol Chem 283:35053-35059.

Marsan E, Baulac S (2018) Review: mechanistic target of rapamycin (mTOR) pathway, focal cortical dysplasia and epilepsy. Neuropathol Appl Neurobiol 44:6-17.
Meng XF, Yu JT, Song JH, Chi S, Tan L (2013) Role of the mTOR signaling pathway in epilepsy. J Neurol Sci 332:4-15.

Mirzaa GM, Campbell CD, Solovieff N, Goold C, Jansen LA, Menon S, Timms AE, Conti V, Biag JD, Adams C, Boyle EA, Collins S, Ishak G, Poliachik S, Girisha KM, Yeung KS, Chung BHY, Rahikkala E, Gunter SA, McDaniel SS, et al. (2016) Association of MTOR mutations with developmental brain disorders, including megalencephaly, focal cortical dysplasia, and pigmentary mosaicism. JAMA Neurol 73:836-845.

Nellist M, Sancak O, Goedbloed MA, Rohe C, van Netten D, Mayer K, Tucker-Williams A, van den Ouweland AM, Halley DJ (2005) Distinct effects of single amino-acid changes to tuberin on the function of the tuberin-hamartin complex. Eur J Hum Genet 13:59-68.

Northrup H, Koenig MK, Pearson DA, Au KS (1999) Tuberous sclerosis complex. Research In: GeneReviews (Adam MP, Ardinger HH, Pagon RA, Wallace SE, Bean LJH, Stephens K, Amemiya A, eds). Seattle, WA: University of Washington.

Orlova KA, Crino PB (2010) The tuberous sclerosis complex. Ann N Y Acad Sci 1184:87-105.

Ostendorf AP, Wong M (2015) mTOR inhibition in epilepsy: rationale and clinical perspectives. CNS Drugs 29:91-99.

Park SM, Lim JS, Ramakrishina S, Kim SH, Kim WK, Lee J, Kang HC, Reiter JF, Kim DS, Kim HH, Lee JH (2018) Brain somatic mutations in MTOR disrupt neuronal ciliogenesis, leading to focal cortical dyslamination. Neuron 99:83-97.e7.

Pun RY, Rolle IJ, Lasarge CL, Hosford BE, Rosen JM, Uhl JD, Schmeltzer SN, Faulkner C, Bronson SL, Murphy BL, Richards DA, Holland KD, Danzer SC (2012) Excessive activation of mTOR in postnatally generated granule cells is sufficient to cause epilepsy. Neuron 75:1022-1034.

Rosset C, Netto CBO, Ashton-Prolla P (2017) TSC1 and TSC2 gene mutations and their implications for treatment in tuberous sclerosis complex: a review. Genet Mol Biol 40:69-79.

Saxton RA, Sabatini DM (2017) mTOR signaling in growth, metabolism, and disease. Cell 169:361-371.

Tavazoie SF, Alvarez VA, Ridenour DA, Kwiatkowski DJ, Sabatini BL (2005) Regulation of neuronal morphology and function by the tumor suppressors Tsc1 and Tsc2. Nat Neurosci 8:1727-1734.

Tsai V, Parker WE, Orlova KA, Baybis M, Chi AW, Berg BD, Birnbaum JF, Estevez J, Okochi K, Sarnat HB, Flores-Sarnat L, Aronica E, Crino PB (2014) Fetal brain mTOR signaling activation in tuberous sclerosis complex. Cereb Cortex 24:315-327.

Vezzani A, Auvin S, Ravizza T, Aronica E (2012) Glia-neuronal interactions in ictogenesis and epileptogenesis: role of inflammatory mediators. In: Jasper's basic mechanisms of the epilepsies (Noebels JL, Avoli M, Rogawski MA, Olsen RW, Delgado-Escueta AV, eds). Bethesda, MD: National Center for Biotechnology Information (US).

Wiegand G, May TW, Ostertag P, Boor R, Stephani U, Franz DN (2013) Everolimus in tuberous sclerosis patients with intractable epilepsy: a treatment option? Eur J Paediatr Neurol 17:631-638.

Wong M, Roper SN (2016) Genetic animal models of malformations of cortical development and epilepsy. J Neurosci Methods 260:73-82.

Zeng LH, Rensing NR, Wong M (2009) The mammalian target of rapamycin signaling pathway mediates epileptogenesis in a model of temporal lobe epilepsy. J Neurosci 29:6964-6972. 